\title{
Incidência de violência física doméstica contra crianças e adolescentes em uma cidade do interior da Bahia durante a pandemia de COVID-19
}

\author{
Incidence of domestic physical violence against children and adolescents in a city in the interior \\ Bahia during the COVID-19 pandemic \\ Incidencia de violencia física domestica contra niños y adolescentes en una ciudad del interior de \\ Bahia durante la pandemia del COVID-19
}

Recebido: 02/12/2021 | Revisado: 08/12/2021 | Aceito: 12/12/2021 | Publicado: 21/12/2021

\section{Resumo}

Jaianne Oliveira Leão Silva

ORCID: https://orcid.org/0000-0002-8741-3128 Faculdade Independente do Nordeste, Brasil E-mail: jaianneleao@gmail.com

Érika Pereira de Souza

ORCID: https://orcid.org/0000-0002-6128-329X

Faculdade Independente do Nordeste, Brasil E-mail:erikasouza@fainor.com.br

Jainne Oliveira Leão Silva

ORCID: https://orcid.org/0000-0002-4796-083X

Faculdade Independente do Nordeste, Brasil

E-mail: jainneleao2015@gmail.com

Igor da Silva Val

ORCID: https://orcid.org/0000-0002-7566-8887 Centro Universitário Faculdade de Tecnologia e Ciência, Brasil E-mail: igorval.800@hotmail.com

Bruno Costa da Silva

ORCID: https://orcid.org/0000-0002-8331-5897

Centro Universitário Maurício de Nassau, Brasil E-mail:brunocosta.yes@gmail.com

Gisele Santos Oliveira Borges

ORCID: https://orcid.org/0000-0002-1017-654X

Centro Universitário Maurício de Nassau, Brasil E-mail: giselesoborges@hotmail.com

Laura Müller

ORCID: https://orcid.org/0000-0002-0759-4898 Centro Universitário Faculdade de Tecnologia e Ciências, Brasil E-mail: lauramullerlima@hotmail.com

Lucas Brito dos Santos

ORCID: https://orcid.org/0000-0002-2747-5730 Instituição de Medicina Hiperbárica, Brasil E-mail:lucasbritorc@gmail.com

Karine de Jesus Silva

ORCID: https://orcid.org/0000-0003-1145-2398

Faculdade Independente do Nordeste, Brasil

E-mail: karinedejesussilva000@gmail.com

Vitor Duarte Santos

ORCID: https://orcid.org/0000-0002-2637-3396 Centro Universitário Maurício de Nassau, Brasil E-mail: vitorduarte.vs@gmail.com

A pandemia de COVID-19 trouxe inúmeros fatores estressantes para a população, ocasionando um aumento da violência contra a criança e adolescente. O objetivo deste trabalho foi verificar a incidência de violência física doméstica contra crianças e adolescentes em uma cidade do interior da Bahia durante a pandemia de COVID-19. Trata-se de um estudo transversal de caráter quantitativo, onde os dados da pesquisa foram obtidos através do Departamento de Informática do Sistema Único de Saúde, provenientes do Sistema de Informação de Agravos de Notificação, para a coleta de dados, foram consideradas as notificações do ano de 2019. A amostra foi composta por 658 casos de violência física doméstica. O principal local de ocorrência foi a residência (357 casos) ocorridos com adolescentes do sexo feminino (368 casos), com idade de 15 a 19 anos (69 casos), de raça/cor parda (198 casos). No que se refere a relação parenteral, 268 casos foram cometidos por pessoas adultas, onde 87 casos foram cometidos pelo ex-cônjuge. Em relação a evolução do caso, apesar de haver tal variável, essas informações foram preenchidas como em branco e ignorado. Foi possível concluir 
com esse estudo que a violência contra crianças e adolescentes cresceu de forma expressiva durante a pandemia de COVID-19, sendo possível observar também a necessidade de trabalho atuais sobre a temática, além disso, é importante salientar a necessidade do correto e completo preenchimento da ficha de notificação.

Palavras-chave: COVID-19; Criança e adolescente; Pandemia; Violência doméstica; Violência infantil.

\begin{abstract}
The COVID-19 pandemic brought numerous stressful factors to the population, causing an increase in violence against children and adolescents. The objective of this work was to verify the incidence of domestic physical violence against children and adolescents in a city in the interior of Bahia during the COVID-19 pandemic. This is a cross-sectional quantitative study, where the research data were obtained through the Information Technology Department of the Unified Health System, from the Information System for Notifiable Diseases, for data collection, the notifications were considered. year 2019. The sample consisted of 658 cases of domestic physical violence. The main place of occurrence was the residence (357 cases) which occurred with female adolescents (368 cases), aged from 15 to 19 years (69 cases), of mixed race/color (198 cases). With regard to parenteral intercourse, 268 cases were committed by adults, where 87 cases were committed by the ex-spouse. Regarding the evolution of the case, despite the existence of such a variable, this information was filled in as blank and ignored. It was possible to conclude with this study that violence against children and adolescents grew significantly during the COVID-19 pandemic, and it is also possible to observe the need for current work on the subject, in addition, it is important to emphasize the need for correct and complete filling in the notification form.
\end{abstract}

Keywords: COVID-19; Child and teenager; Pandemic; Domestic violence; Child Violence.

\title{
Resumen
}

La pandemia COVID-19 trajo numerosos factores estresantes a la población, provocando un aumento de la violencia contra niños y adolescentes. El objetivo de este trabajo fue verificar la incidencia de violencia física doméstica contra niños, niñas y adolescentes en una ciudad del interior de Bahía durante la pandemia Covid-19. Se trata de un estudio cuantitativo transversal, donde los datos de la investigación se obtuvieron a través del Departamento de Informática del Sistema Único de Salud, del Sistema de Información de Enfermedades Notificables, para la recolección de datos, se consideraron las notificaciones. Año 2019, la muestra estuvo conformada por 658 casos de violencia física doméstica. El principal lugar de ocurrencia fue la residencia (357 casos) que ocurrió con mujeres adolescentes (368 casos), de 15 a 19 años (69 casos), mestizas / color (198 casos). En cuanto al coito parenteral, 268 casos fueron cometidos por adultos, de los cuales 87 fueron cometidos por el excónyuge. En cuanto a la evolución del caso, a pesar de la existencia de dicha variable, esta información se completó en blanco y se ignoró. Se pudo concluir con este estudio que la violencia contra la niñez y adolescencia creció significativamente durante la pandemia del COVID-19, y también es posible observar la necesidad de un trabajo actual en el tema, además, es importante enfatizar la necesidad de cumplimentación correcta y completa del formulario de notificación.

Palabras clave: COVID-19; Niño y adolescente; Pandemia; La violencia doméstica; Violencia infantil.

\section{Introdução}

Em dezembro de 2019, foi noticiado pela imprensa mundial a existência de um vírus que havia se proliferado, em seguida foi confirmado o primeiro caso da COVID-19, na cidade de Wuhan, na China (Marques et al., 2020).

O vírus se propagou por diversos países, transformando-se em uma pandemia, afetando o mundo de uma forma peculiar, alterando as relações econômicas, políticas e sociais (França; Ferreira, 2020). Os autores ainda pontuam que a pandemia do COVID-19 tem sido devastadora na América Latina e Caribe.

De acordo com Teixeira (2020), a pandemia no Brasil se dividiu em dois momentos, o primeiro foi o momento em que pessoas de classe alta e média que viajaram para a Europa ou Estados Unidos da América contraíram a doença e disseminaram o vírus no seu ciclo de amizade, familiares e empregados, já o segundo momento foi de expansão da doença, resultante da transmissão comunitária.

Segundo Moraes (2020) a pandemia de COVID-19 trouxe inúmeros fatores estressantes para a população, fatores esses que não existiam em períodos de normalidade. Alguns desses fatores resultam da própria pandemia, enquanto outros, de suas políticas de enfrentamento.

Na cartilha elaborada pelo Ministério da Saúde, intitulada:” Crianças na pandemia COVID-19” expõe que, no cenário da pandemia, as autoridades adotaram medidas sanitárias para conter a rápida disseminação do contágio da COVID-19, 
destacando como principal medida o distanciamento social, implicando no fechamento de escolas, interferindo na rotina e nas relações interpessoais das crianças e dos adolescentes (Brasil, 2020).

Mata e colaboradores (2021) apontam que apesar de benéfico à saúde, o distanciamento social para as crianças e para os adolescentes podem causar consequências psicológicas a longo prazo, incluindo transtorno de estresse pós-traumático, ansiedade, depressão e até tendência suicida, os autores explicam que o contato interpessoal é importantíssimo para o ser humano, sendo um componente fundamental para o desenvolvimento neuropsicológico adequado das crianças e dos adolescentes.

Moreira e Magalhães (2020) reforçam dizendo que a dinâmica do ambiente intrafamiliar é preocupante dentro do contexto de isolamento social causado pela pandemia de COVID-19, pois a violação dos direitos das crianças e adolescentes tendem a ser agravado em decorrência da mudança de comportamento das pessoas que compartilham o mesmo domicilio, onde as mesmas ainda acabam ficando cada vez mais expostas a situações de violência no ambiente familiar.

Mores (2020) cita alguns fatores estressantes ocasionados pela pandemia, como o próprio confinamento, a diminuição da renda, o medo de ser infectado ou de alguém próximo se infectar, e as informações imprecisas sobre a pandemia e sobre o enfrentamento da mesma.

Em conformidade com o Estatuto da Criança e do Adolescente (ECA), é considerado criança, a pessoa até doze anos de idade incompletos, sendo que a atualização mais recente da OMS considera criança a pessoa na faixa etária de zero a 9 anos (BRASIL, 1990; BRASIL, 2018). Já a definição de adolescência é prescrita pela Organização Mundial da Saúde (OMS), caracterizando-se pelo período entre 10 a 19 anos (Brasil, 2018).

No ECA (1990) é declarado que nenhuma criança ou adolescente poderá ser alvo de qualquer forma de negligência, discriminação, exploração, crueldade e opressão, punido pela lei qualquer atentado, ação ou omissão, aos seus direitos. Poletto (2016) demonstrou que a infância e a adolescência constituem fases de desenvolvimento continuo e de intenso processo de maturação, onde as experiências vividas nessas etapas podem deixar registros, e mesmo sendo capazes de serem reparadas ou reconstruídas, costumam deixar marcas importantes.

Contudo, muitas crianças experienciam a violência, que, segundo Melo et al. (2016), é conceituada como um fenômeno complexo, manifestando-se de diversas formas, em diferentes contextos, sendo potencialmente capaz de causar danos e afetar a saúde individual e coletiva dos envolvidos. No Relatório Mundial sobre a Violência e a Saúde, a Organização Mundial de Saúde (OMS) define o evento violento como: o uso intencional da força física ou do poder, sendo de forma real ou por ameaça, contra a própria pessoa, outra pessoa, um grupo ou comunidade, podendo resultar em morte, lesão, dano psicológico, problemas de desenvolvimento ou de privação (Sousa et al., 2013).

O ato de violência que ocorre em casa, é intitulado de violência doméstica (Melo et al., 2016). Segundo Pita (2015), a condição doméstica que envolve a família é configurada como um ambiente reservado, onde as relações entre seus integrantes se estabelecem em seu interior enquanto espaço privado. É importante destacar que a violência doméstica não é um acontecimento exclusivo das classes menos favorecidas, pode acontecer e atinge famílias de todas as classes sociais, abstraindo de raça, religião ou nível intelectual. No entanto, os episódios de violência se tornam mais evidentes nas classes menos favorecidas (Brasil; Penna, 2004).

É um fato que a violência contra crianças e adolescentes seja um problema de saúde pública global e que os fatores geográficos e econômicos são capazes de influenciar as variações nas estimativas de violência, no entanto, existem outros fatores que podem aumentar a susceptibilidade para a violência infantil, como a austeridade econômica, desastres e emergências (Levandowsky et al., 2021). Tendo em vista a identificação do elevado número de agressão, a autora Poletto (2016) indica que $\mathrm{o}$ ato de violência ocupa atualmente o status de epidemia.

Pita (2015) sustenta que o evento da violência contra a criança e adolescente envolve três aspectos, o primeiro deles é o aspecto interpessoal, em que o adulto com seu poder aprisiona a vontade e o desejo da criança, o segundo aspecto é o social, 
de causa estrutural com a existência da desigualdade social, e terceiro aspecto é o sociointeracionista, apoiando a ideia de que o indivíduo aprende na interação com o outro, reforçando que a família tem um papel importante na formação da criança e a escola na educação do cidadão, promovendo a ruptura do ciclo de violência.

O Estatuto da Criança e do Adolescente (1990) apresenta um documento com legislações avançadas e atualizadas, cabendo ressaltar aqui a citação que o ECA faz sobre a Lei do menino Bernardo (lei no 13.010, de 26 de junho de 2014), onde a mesma estabelece o direito da criança e do adolescente de serem educados sem o uso de castigos físicos ou de tratamento cruel ou degradante. Concluindo esse pensamento, o ECA afirma que a criança e o adolescente têm o direito de ser educados através de formas de correção, disciplina e educação.

Por observar um número reduzido de estudos que analisam a violência doméstica em crianças e adolescentes durante a pandemia de COVID 19, torna-se necessário a execução deste estudo, visto que o mesmo se insere no contexto atual como uma contribuição ao debate sobre o cenário de risco que essa violência pode ocorrer, como o isolamento social. Desta forma, este estudo irá contribuir para identificar a incidência da violência doméstica em crianças e adolescentes e, a partir dos seus resultados, possibilitar a criação de estratégias para o enfrentamento da violência por gestores da saúde e atores sociais, o objetivo do estudo é verificar a incidência de violência doméstica contra crianças e adolescentes em uma.

\section{Metodologia}

Trata-se de um estudo transversal de caráter quantitativo. Os estudos transversais são considerados como uma valiosa ferramenta e de grande utilidade para a descrição de característica da população, sendo utilizada para a identificação de grupos de risco e para a ação e o planejamento em saúde. Quando utilizados de acordo com suas indicações, vantagens e limitações, podem oferecer valiosas informações para o avanço do conhecimento científico (BASTOS; DUQUIA, 2007).

Já o caráter quantitativo tange ao que pode ser quantificado, com o propósito de investigar fatos, aspectos e fenômenos. Esse tipo de abordagem se preocupa com a precisão cientifica, utilizando-se de recursos formais, como por exemplo, as técnicas estatísticas, conhecidas pela precisão e controle das informações coletadas e analisadas (SILVA, 2018).

Os dados foram obtidos através do Departamento de Informática do Sistema Único de Saúde (DATASUS), provenientes do Sistema de Informação de Agravos de Notificação (SINAN). A população do estudo foi constituída por 658 casos de violência física doméstica, registrados no período de 2019, ocorridas em uma cidade do interior da Bahia.

Por critério de inclusão, foram considerados: sexo feminino e masculino; faixa etária foi categorizada em: $<1$ ano, 1 a 4 anos, 5 a 9 anos, 10 a 14 anos, 15 a 19 anos e ignorado/em branco. A variável cor/raça foi categorizada em: branca, preta, amarela, parda, indígena e ignorado/em branco; o local da ocorrência foi a residência, o tipo de violência selecionados foi a violência física, o ciclo de vida do agressor selecionado foi: pessoa adulta, pessoa idosa e ignorado/em branco, as relações parenterais selecionadas foram: pai, mãe, padrasto, madrasta, conjugue, ex-cônjuge, cuidador e a evolução do caso resultando em Óbito por violência, Ignorado/ Em branco.

Foram excluídos dados como violência física doméstica ocorrida em pessoas acima de 19 anos, no ciclo de vida do autor foram excluídas as variantes: criança, adolescente e jovem, também foi excluído os números que aparecem como critério "não" após filtragem de dados.

Os dados foram organizados de forma que fossem consideradas as notificações do ano de 2019, ano onde houve o início da pandemia de COVID-19.

A pesquisa obedeceu aos aspectos éticos e legais dispostos na Lei $\mathrm{n}^{\circ} 9.610$ do dia 19 de fevereiro de 1998 sobre plágio e direitos autorais, assegurando que todos os autores consultados fossem referenciados. 


\section{Resultados}

Compuseram a amostra desta pesquisa 658 casos de violência física doméstica. Desse número, 130 crianças e adolescentes eram do sexo masculino e 528 do sexo feminino.

Houve 75 casos de violência contra meninos, 368 casos de violência contra meninas e 9 casos foram ignorados.

Com relação a faixa etária, houve 1 caso de violência contra criança abaixo de 1 ano de idade, 8 casos em crianças com idade entre 1 a 4 anos, 11 casos de violência física domestica em criança de 5 a 9 anos, 30 casos em crianças de 10 a 14 anos, 69 casos em adolescentes de 15 a 19 anos e 4 em casos a faixa etária não foi descrita.

No que diz respeito a raça, 51 crianças brancas foram vítimas de violência física doméstica, 65 crianças da raça preta, 2 crianças da raça amarela, 198 crianças da raça parda, 3 crianças da raça indígena e 9 sem a identificação de da raça.

Quanto ao local de ocorrência, houveram 357 casos ocorridos na residência e 5 casos foram ignorados.

No que se refere ao ciclo de vida do autor, houve 268 casos cometidos por pessoas adultas, 12 casos cometidos por pessoa idosa e 4 casos sem a identificação do ciclo do autor.

No que concerne a relação parenteral do autor com a vítima, 87 casos foram cometidos pelo ex-cônjuge, 66 casos foram cometidos pelo conjugue, 24 casos foram cometidos pelo pai, 13 casos foram cometidos pela mãe, 8 casos foram cometidos pelo padrasto, 1 caso foi cometido pela madrasta, e 1 caso foi cometido pelo (a) cuidador (a).

Em relação a evolução do caso, apesar de haver tal variável, essas informações foram preenchidas como em branco e ignorado.

\section{Discussão}

Uma pesquisa realizada pelo IBGE, em 2012, indicou que adolescentes com idades entre 13 e 15 anos já haviam sofrido agressão física por um adulto da família, onde 11,5\% eram meninas e 9,6\% eram meninos (Brasil, 2018). Souto e colaboradores (2017) afirmam que é extremamente importante diferenciar qual sexo sofre mais com esse tipo de violência. No presente estudo, foi possível observar que o sexo feminino liderou os casos de violência física doméstica, entre os 528 casos,

Entre 2011 e 2016, a Vigilância de Violências e Acidentes (Viva) notificou um aumento considerável de casos de violências contra crianças de zero a 9 anos. No ano de 2016, foram registrados 40.569 casos de violências contra crianças, sendo 22.076 contra meninas e 18.439 contra meninos (Brasil, 2018). Em outros estudos também foi encontrado resultados parecidos, onde o sexo feminino apresentou maior proporção de vítimas de violência física em relação ao sexo masculino (Levandowski, 2021; Martins; Jorge, 2009).

No entanto, segundo o Ministério da Saúde (2012), os meninos sofrem mais agressões físicas, enquanto as meninas estão mais expostas aos tipos de violência como: sexual, negligência nutricional e educacional e exploração sexual comercial. O mesmo foi identificado por Barker, de que crianças e adolescentes do sexo masculino são as maiores vítimas de abuso físico ou maus tratos físicos em suas casas, enquanto as meninas sofrem mais com a violência e o abuso sexual (2008). Já em Uganda, houve $68 \%$ de casos de violência física contra meninos e $55 \%$ em meninas e na Costa Rica $52 \%$ para meninos e $39 \%$ para meninas, sendo possível observar que o sexo masculino foi predominante (Simões, Athaias e Botelho, 2018).

Sobre a faixa etária, o trabalho presente evidenciou que o maior número de violência física doméstica, com 69 casos, foi contra adolescentes de 15 a 19 anos de idade, sendo possível observar que essa faixa etária também foi predominante no estudo de Poletto (2016), evidenciando que na cidade de Concórdia- SC, houveram 5.331 casos de violência contra adolescentes na faixa etária entre 15 e 19 anos.

No entanto, Peres e coadjutores (2015) afirmam que a violência domiciliar é mais frequente na primeira infância, onde são consideradas crianças abaixo de 5 anos, geralmente provocada por parte de seus cuidadores, mas pode ser também demais 
membros da família, em razão de sua dependência e limitadas interações sociais fora do círculo doméstico. No documento intitulado: "Letalidade infanto-juvenil: dados da violência e políticas públicas existentes", a idade mais atingida foi de 4 a 11 anos com $40 \%$, seguindo a faixa etária de 12 a 17 anos com $31 \%$, e de 0 a 3 anos com $16 \%$.

No documento elaborado pelo Ministério dos Direitos Humanos (2018), a raça/cor obteve 57,5\% meninas e meninos negros/pardos vítimas de violência física doméstica. Já o Ministério da Saúde (2018) evidenciou 39,3 \% de casos com a raça/cor branca e 37,4\% parda. No que diz respeito a raça, o maior número encontrado no estudo presente foram 198 crianças, da raça/cor parda.

Os autores Platt, Guedert e Coelho (2020) afirmam que o local mais frequente das ocorrências de violência, é a casa da vítima. Local onde deveria ser mais seguro para as pessoas estarem protegidas, pode ser o local mais inseguro para muitas crianças e adolescentes. Quanto ao local de ocorrência, neste estudo 357 casos foram ocorridos na residência. Sendo esse fato confirmado pelo Ministério da Saúde (2012) que a residência foi o local de maior ocorrência dos casos de violência contra crianças.

A origem do lar como local de episódios violentos, revela que os agressores são da família ou têm livre acesso à residência (Souto et al, 2018). Assim como no estudo de Martins e Jorge (2009) onde a maior parte dos agressores era do sexo masculino, sendo o pai, o principal agressor por força corporal. Já o Ministério dos Direitos Humanos (2018) expõe a mãe, como principal suspeita de agressões, em seguida aparece o pai, o padrasto e a avó.

Dentro dessa assertiva, o estudo presente evidencia 268 casos cometidos por pessoas adultas e 87 dos casos foram cometidos pelo ex-cônjuge.

Melo e outros autores (2016) concordam com essa asserção e amplificam a informação, acrescentando o desemprego, a baixa escolaridade, a falta de planejamento familiar e a pobreza, como motivos que concorrem para a ocorrência da violência contra crianças e adolescentes.

Peres e coadjutores (2015) elegem inúmeros fatores que podem influenciar para a ocorrência do ato de violência, alguns desses fatores são: as características da vítima e o contexto no qual a mesma está inserida, da sua família, do agressor e as características de forma geral da sociedade, esses fatos propiciam e, consequentemente, contribuem para a vulnerabilidade à violência infantil.

De acordo com o Ministério da saúde (2018), a violência/agressão, embora seja frequente, nem sempre leva a internação hospitalar ou ao óbito. Um estudo demonstra que em 2007, as agressões ocupavam a quinta causa de óbitos de crianças menores de um ano de idade, no Brasil (Brasil, 2012).

Se tratando da evolução do caso, 443 casos não tiveram a evolução informada no momento da notificação. Em outro documento, o Ministério da Saúde adiciona que, mesmo a maior parte das vítimas de violência sejam não-letais e não evoluem a óbito, ainda sim esses atos podem causar severos danos à saúde física e mental (Brasil, 2010).

Com o distanciamento social, Mata e colaboradores (2020) confirmam a diminuição do sistema de apoio durante o período de pandemia, somado a exposição à eventos estressores, que exacerbam a vulnerabilidade à violência doméstica contra crianças e adolescentes.

Souto e colaboradores (2018) afirmam que a notificação compulsória da violência deve ser compreendida como uma ferramenta importantíssima, onde é possível garantir os direitos e proteção social de crianças e adolescentes, possibilitando ainda aos profissionais de saúde, assim como da educação, da assistência social, dos Conselhos Tutelares e da justiça, adotarem medidas de cuidado às vítimas da violência. 


\section{Conclusão}

Com esse trabalho, obteve-se importantes informações acerca da violência física doméstica á crianças e adolescentes, tal como um aumento expressivo desses casos durante a pandemia de COVID-19, evidenciando a necessidade de mais estudos científicos atuais sobre a temática, bem como incentivar e orientar sobre importância da notificação, sendo de extrema importância para intervir e prevenir ato de violência física doméstica.

Torna-se importante ressaltar a necessidade do correto e completo preenchimento da ficha de notificação, pois foi possível observar que muitas das informações pertinentes ao preenchimento das fichas analisadas estiveram ausentes ou incompletas, verificando-se a fragilidade profissional quanto ao manejo das fichas, percebida em deficiência de conhecimento e de responsabilidade para com o preenchimento.

Desta forma, este estudo contribui para permitir um maior conhecimento da população e da comunidade cientifica sobre a temática, favorecendo assim, políticas sociais e de saúde para crianças e adolescentes no intuito de favorecer proteção e suporte a esse público.

\section{Referência}

Barros, A. S. D., \& Freitas, M. D. F. Q. D. (2015). Violência doméstica contra crianças e adolescentes: consequências e estratégias de prevenção com pais agressores. Pensando famílias, 19(2), 102-114.

Conquista, V. (2015). Memória e Infância: manifestações da Violência Doméstica nos Anos Escolares.

Silva, L. M. P. D., Ferriani, M. D. G. D. C., \& Silva, M. A. I. (2011). Atuação da enfermagem frente à violência sexual contra crianças e adolescentes. Revista Brasileira de Enfermagem, 64, 919-924.

Mata, A. A., Lana, A. C. F., de Souza Bernardes, F., de Araújo Gomes, G., Silva, I. R., Meirelles, J. P. S. C., ... \& de Souza Bechara, L. (2021). Impacto da pandemia de COVID-19 na saúde mental de crianças e adolescentes: uma revisão integrativa. Brazilian Journal of Development, 7(1), 6901-6917.

Rosa Moreira, R. B., \& de Oliveira Magalhães, D. K. (2020). Os impactos da pandemia de covid-19 no enfrentamento da violência sexual intrafamiliar contra crianças e adolescentes. Revista da Jornada de Pós-Graduação e Pesquisa-Congrega Urcamp, 16, $269-275$.

Macêdo, V. C. (2016). Atenção integral à saúde da criança: políticas e indicadores de saúde.

Cintra, J. P. S., \& Mathias, R. (2018). Cenário da Infância e Adolescência no Brasil 2019. São Paulo: Fundação Abrinq.

Melo, R. A., de Lima Souza, S., Bezerra, C. S., \& Fernandes, F. E. C. V. (2016). Assistência de enfermagem a criança e ao adolescente em situação de violência doméstica. ID on line Revista de Psicologia, 10(32), 245-259.

Mesquita, E. A. (2020). A responsabilidade social do empregador durante a pandemia da covid-19. Ponto de Vista Jurídico, 60-77.

Eisenstein, E. (2005). Adolescência: definições, conceitos e critérios. Adolescência e saúde, 2(2), 6-7.

Justino, L. C. L., Nunes, C. B., Gerk, M. A. D. S., Fonseca, S. S. O., Ribeiro, A. A., \& Paranhos, A. C. (2015). Violência sexual contra adolescentes em Campo Grande, Mato Grosso do Sul. Revista Gaúcha de Enfermagem, 36, 239-246.

Levandowski, M. L., Stahnke, D. N., Munhoz, T. N., Hohendorff, J. V., \& Salvador-Silva, R. (2021). Impacto do distanciamento social nas notificações de violência contra crianças e adolescentes no Rio Grande do Sul, Brasil. Cadernos de Saúde Pública, 37.

Linhares, MBM e Enumo, SRF (2020). Reflexões baseadas na Psicologia sobre efeitos da pandemia COVID-19 no desenvolvimento infantil. Estudos de Psicologia (Campinas), 37 .

Lopez, S. B., \& Moreira, M. C. N. (2013). Políticas Nacionais de Atenção Integral à Saúde de Adolescentes e Jovens e à Saúde do Homem: interlocuções políticas e masculinidade. Ciência \& Saúde Coletiva, 18, 743-752.

Marques, E. S., Moraes, C. L. D., Hasselmann, M. H., Deslandes, S. F., \& Reichenheim, M. E. (2020). A violência contra mulheres, crianças e adolescentes em tempos de pandemia pela COVID-19: panorama, motivações e formas de enfrentamento. Cadernos de Saúde Pública, 36, e00074420.

Martins, CBDG, \& Jorge, MHPDM (2009). Violência física contra menores de 15 anos: estudo epidemiológico em cidade do sul do Brasil. Revista brasileira de epidemiologia , 12 , 325-337.

Melo, B. D., Lima, C. C., Moraes, C. L. D., Andrade, C. B., Pereira, D. R., Souza, E. R. D., ... \& Freitas, C. M. D. (2020). Saúde mental e atenção psicossocial na pandemia COVID-19: violência doméstica e familiar na COVID-19.

Ministério dos Direitos Humanos. Secretaria Nacional de Proteção dos Direitos da Criança e Adolescente. Letalidade infanto-juvenil: dados da violência e políticas públicas existentes / elaboração de Thaís Cristina Alves Passos - Documento eletrônico - Brasília: Ministério dos Direitos Humanos, 2018, 110 p. 
Research, Society and Development, v. 10, n. 17, e47101724287, 2021

(CC BY 4.0) | ISSN 2525-3409 | DOI: http://dx.doi.org/10.33448/rsd-v10i17.24287

Moraes, R. F. D. (2020). Prevenindo conflitos sociais violentos em tempos de pandemia: garantia da renda, manutenção da saúde mental e comunicação efetiva.

Nunes, M. C. A., Lima, R. F. F., \& Morais, N. A. D. (2017). Violência sexual contra mulheres: um estudo comparativo entre vítimas adolescentes e adultas. Psicologia: ciência e profissão, 37, 956-969..

ORR, Carol et al. A exposição à violência familiar e doméstica está associada ao aumento das hospitalizações na infância. PLoS one , v. 15, n. 8, pág. e0237251, 2020 .

Paixão, ACWD, \& Deslandes, SF (2010). Análise das políticas públicas de enfrentamento da violência sexual infantojuvenil. Saúde e Sociedade , 19 (1), 114126.

Peres, M. F. T., Ruotti, C., Carvalho, D., \& Regina, F. L. (2015). Vitimização fatal de crianças no espaço público em decorrência da violência interpessoal comunitária: um diagnóstico da magnitude e contextos de vulnerabilidade na América Latina. Revista Brasileira de Segurança Pública, 9(2).

Poletto, L. T. (2016). Percepções de adolescentes que frequentam o $9^{\text {a }}$ ano do ensino fundamental de uma escola do município de Concórdia sobre o tema "violência contra crianças e adolescentes".

Santa Rita, A. C. M., de Castro, A. C. G., Roberti, B. N., Teixeira, I. D., de Menezes, L. B. R., Tavares, M. B., ... \& Grillo, C. D. F. C. (2020). Violência infantojuvenil intrafamiliar e doméstica: o impacto do distanciamento social e a importância da conscientização em meio à pandemia de COVID-19. Revista Eletrônica Acervo Saúde, 12(10), e4689-e4689.

Sawyer, S. M., Azzopardi, P. S., Wickremarathne, D., \& Patton, G. C. (2018). A idade da adolescência. The Lancet Child \& Adolescent Health , 2 (3), 223-228.

Silva, E. R. A. D., \& Oliveira, V. R. D. (2020). Proteção de crianças e adolescentes no contexto da pandemia da Covid-19: consequências e medidas necessárias para o enfrentamento.

Silva, S. N. (2016). Desvendando a violência sexual contra crianças e adolescentes no município de Vitória da Conquista.

Simões, A., Athias, L., \& Botelho, L. (Eds.). (2018). Panorama nacional e internacional da produção de indicadores socias: grupos populacionais específicos e uso do tempo. IBGE, Instituto Brasileiro de Geografia e Estatística.

Sousa, N. S., Vieira, C. S., Fernandes, P. A., \& Sousa, C. S. (2013). Violência doméstica infantil e as políticas públicas. Cadernos da FUCAMP, 12(16).

Souto, D. F., Zanin, L., Ambrosano, G. M. B., \& Flório, F. M. (2018). Violence against children and adolescents: profile and tendencies resulting from Law 13.010. Revista brasileira de enfermagem, 71, 1237-1246. 